\title{
Design and test of irradiation-related components in ITER radial
}

\section{x-ray camera}

L. $\mathrm{Hu}^{1}$, H. Cao ${ }^{1}$, J. Zhao ${ }^{1}$, L. Niu ${ }^{1}$, Y. Chen ${ }^{1}$, N. Zhou ${ }^{1}$, J. Zhan ${ }^{1}, \mathrm{H} . \mathrm{Yu}^{1}$, K. Chen ${ }^{1}, \mathrm{~S}$.

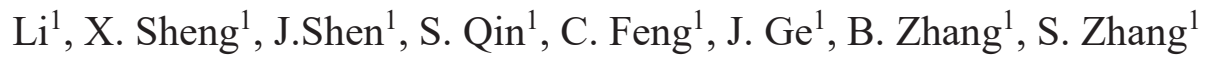

${ }^{1}$ Institute of Plasma Physics, Chinese Academy of Sciences, Hefei 230031, China

E-mail contact of main author: lqhu@ipp.ac.cn

ITER Radial X-ray Camera is designed to measure the poloidal profile of the plasma x-ray emission with high spatial and temporal resolution and will be installed inside equatorial port 12 [1-2]. Overall view of RXC is shown in Fig.1.

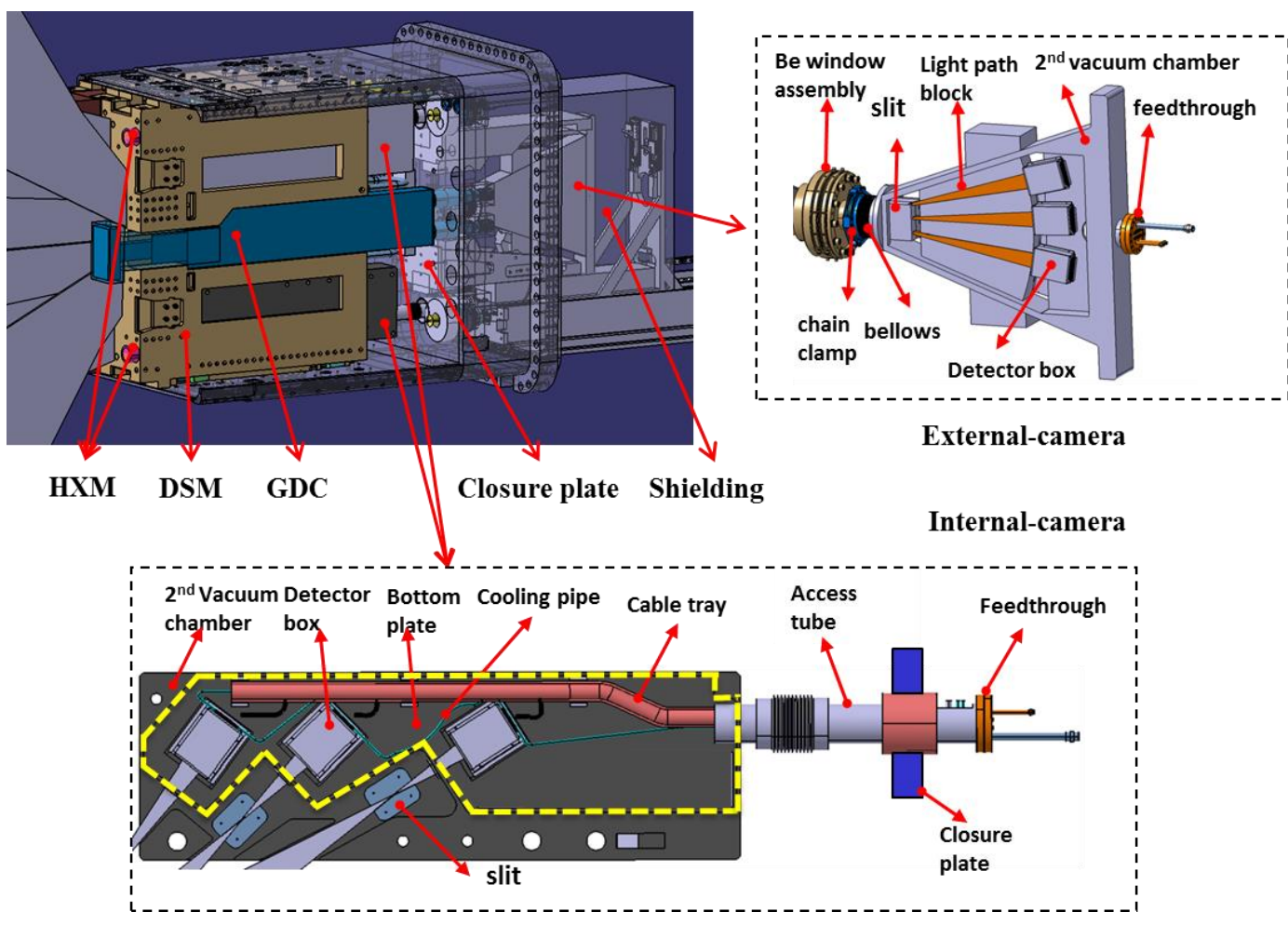

Fig.1. Overall view of $R X C$

As can be seen from Fig.1, RXC, Glow Discharge Cleaning (GDC) and Hard X-ray Monitor (HXM) share middle module of Equatorial 12 port plug [3]. RXC is composed of in-port and ex-port cameras which view the outer and core region respectively through vertical slots in the Diagnostics Shield Module (DSM). A concrete shielding shell enclosing the external camera is designed to reduce radiation leakage into outside environment. 
Due to harsh environment of neutron and gamma radiation, nuclear radiation hardness of many components has to be considered in design and test, including silicon detector array, electronics, cabling, and also remote handling during maintenance. To protect detector against neutron damage, boron carbide blocks are envisaged to put in front of and around detectors to mitigate neutron flux onto detector. The neutron flux onto detectors was calculated. The neutron flux onto internal camera detector in DD operation is in the order of $10^{7} \mathrm{n} / \mathrm{cm}^{2} / \mathrm{s}$, which means the silicon diode detector can survive DD operation without detector replacement [4]. As to external camera, it is possible the silicon detector can survive the whole DT operation with optimized shielding. To guarantee detector survival and performance, advanced detector which should be radiation-hard is being developed. Apart from neutron damage to detector, another important factor to camera design is maintenance under the condition of activation, especially during DT phase. Considering this factor, remote handling strategy has been worked out based on ITER remote handling procedure and tooling.

As for electronics put in port cell, preliminary design of highly integrated pre-amplifier and program controllable mid-amplifier has been completed, and many tests have been done to investigate the radiation hardness performance of the amplifiers together with detectors and cables, although shielding cabinet has been designed to house and enhance the survivability of electronics. In the Cf-252 neutron test with flux of $\sim 2.23 \times 10^{3} \mathrm{n} / \mathrm{cm}^{2} / \mathrm{s}$ and fluence greater than $1.0 \times 10^{10} \mathrm{n} / \mathrm{cm}^{2}$, there was no performance degradation in detector and pre-amplifier. In the Co-60 gamma test with experimental setup shown in Fig.2, under gamma dose rate of $0.5 \mathrm{~Gy} / \mathrm{min}$ and integrated dose of 200Gy, no performance change was found in pre-amplifier and mid-amplifier except for some radiation damage to the power module in the board of pre-amplifier.

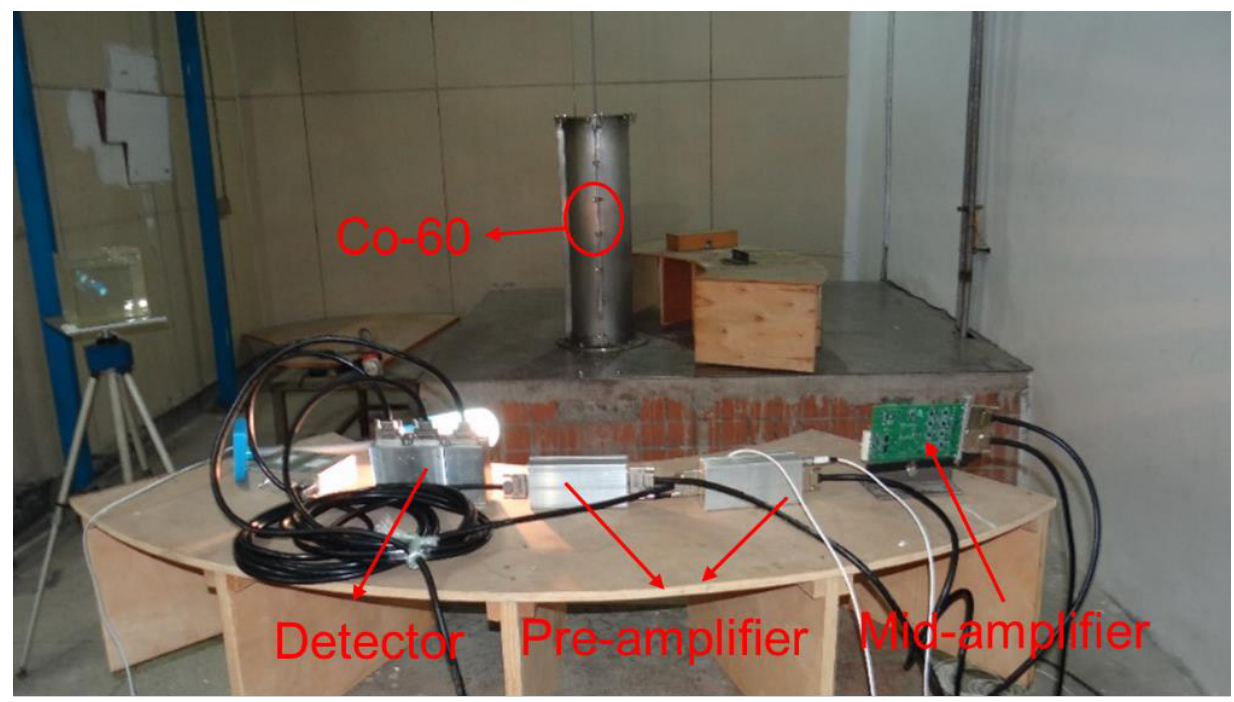

Fig.2. Experimental setup for gamma test

In subsequent accelerator neutron test using 14.9 MeV D-T neutron source where the neutron flux is $\sim 5 \times 10^{7} \mathrm{n} / \mathrm{cm}^{2} / \mathrm{s}$ and neutron fluence $2 \times 10^{12} \mathrm{n} / \mathrm{cm}^{2}$, no function problem was found in pre-amplifier and cable, while there were occurrences of single event effect in mid-amplifier. The detector underwent 10 percent decrease of sensitivity to visible light. The design optimization of 
electronics and further neutron test in reactor will be carried out in the future to improve performance of radiation hardness and qualify the design.

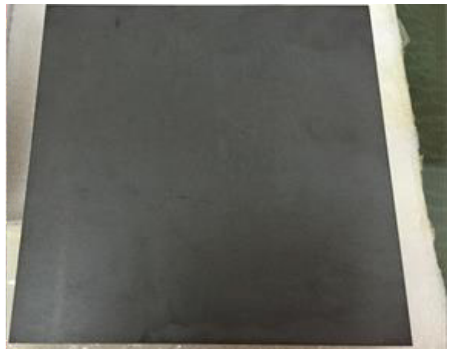

(a)

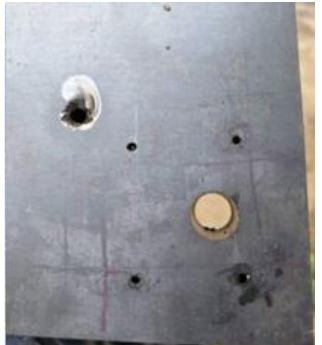

(b)

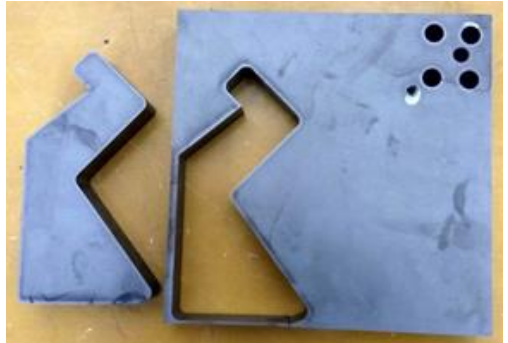

(c)

Fig.3 Manufacturability study of B4C plate. (a) Raw B4C plate; (b) after EDM hole processiong; (c) after wire EDM.

In addition, Manufacture study of $\mathrm{B} 4 \mathrm{C}$ plate and tests were carried out to assess the feasibility of using B4C plate as shielding material. Fig.3 shows the raw B4C plate and those after machined. To produce hole with irregular shape inside B4C plate as shown in Fig.3 (c), the raw B4C plate was firstly drilled rough circular holes with Electrical Discharge Machining (EDM) method, and then cut with wire EDM method. The processed sample surface roughness can be as low as 1.6, making it promising to act as boundary of light of sight or to reduce assembly tolerance.

\section{References}

[1] A. E. COSTLEY, et al., Measurement Requirements and the Diagnostic System on ITER:

Modifications Following the Design Review, in: 22nd IAEA Fusion Energy Conference, Geneva, Swiss, 2008.

[2] S. WANG, et al., Electromagnetic behavior on ITER radial soft x-ray camera, in: 2013 IEEE 25th Symposium on Fusion Engineering (SOFE), San Francisco, United States, 2013.

[3] G. VAYAKIS, et al., Current status of ITER diagnostics development, in:26th ITPA Spring Meeting, Pohang, Korea, 2014.

[4] B. ALPER, et al., The JET soft x-ray diagnostic systems, Rev. Sci. Instrum.,68 (1997) 778. 